DOI: $10.4274 /$ jarem.galenos.2020.3773

J Acad Res Med 2021;11(1):17-23

\title{
Comparison of Feto-maternal Effects of Twin Pregnancies and Twin Pregnancies Caused By Assisted Reproductive Technology
}

\author{
(1) Mustafa Doğan Özçil \\ Hatay Mustafa Kemal University Faculty of Medicine, Department of Obstetrics and Gynecology, Hatay, Turkey \\ Cite this article as: Özçil MD. Comparison of Feto-maternal Effects of Twin Pregnancies and Twin Pregnancies Caused By Assisted Reproductive \\ Technology.J Acad Res Med 2021;11(1):17-23
}

\begin{abstract}
Objective: Comparison of the effects of twin pregnancies using assisted reproductive technology (ART) and spontaneous twin pregnancies on maternal-fetal parameters.

Methods: A total of 5,670 patients who were deliveried in our clinic between 01.01 .2011 and 31.12.2017 were analyzed retrospectively. The fetomaternal results of 94 mothers who deliveried to twins and their infants were compared.

Results: Of the 5,670 patients who gave birth, 94 were twins $(1 / 60)$ and 8 were triplets $(1 / 709)$. Twin births account for $92 \%$ of multiple births. The incidence of spontaneous twin pregnancy was $1 / 77$ and constituted $79 \%$ of all the twin deliveries. The effect of ART on was found to be $21 \%$ [17\% in vitro fertilization (IVF), 4\% ovulation induction].

Mean maternal age was $29.25 \pm 6.00$ years in twin pregnancy deliveries, $28.50 \pm 6.09$ years in mothers who had spontaneous twin group, and $32.54 \pm 5.09$ years in twin births due to ART.

When anemia was excluded, maternal morbidity was $27 \%$ in twin pregnancy deliveries. Pre-eclampsia was determined in $19 \%$ of the cases and anemia in 63\%. Delivery before 37 gestational weeks was recorded in $72.3 \%$ of IVF twins and $70.2 \%$ of spontaneous twins. The rate of live births was found to be higher in spontaneously conceived twins ( $p=0.004)$. The fetal mortality rate was $5.4 \%$ in spontaneous twin pregnancies and $20 \%$ in twin pregnancies with ART ( $p=0.003$ ). The perinatal mortality index was determined to be 81 in spontaneously conceived twins, and 250 in the twin pregnancies obtained with ART $(p=0.006)$.

Conclusion: Premature birth and fetal mortality rates are higher in twin pregnancies obtained with ART than in spontaneous twins. The mortality associated with multiple pregnancies may be reduced with preventive measures such as low-dose gonadotropin use, single embryo transfer, and multifetal embryo reduction.
\end{abstract}

Keywords: Spontaneous pregnancy, assisted reproductive technology, multiple pregnancy

\section{INTRODUCTION}

The pregnancy rate using assisted reproductive technology (ART) has increased significantly in the last four decades. Although ART usage rates in infertile patients are reported as $11 \%$ in the United States of America (USA), this rate is estimated to be higher (1). Twin pregnancy rates due to ART have been reported as $18 \%$ in the USA (1). After ART applications had their place in reproductive biology clinical practice, the incidence of twin birth increased up to $1-4 \%(1-6)$. Although the increase in pregnancy rates was pleasing at first, the significant increase in multiple pregnancy rates due to success-oriented practices such as (i) advanced age, (ii) high dose drug use, (iii) multiple embryo transfer, both

ORCID ID of the author: M.D.Ö. 0000-0003-0819-6733.

Corresponding Author/Sorumlu Yazar: Mustafa Doğan Özçil, E-mail: m.d.ozcil@hotmail.com
Received Date/Geliş Tarihi: 17.09.2020 Accepted Date/Kabul Tarihi: 23.12.2020

(C) Copyright 2021 by University of Health Sciences Turkey, Gaziosmanpaşa Training and Research Hospital. Available on-line at www.jarem.org 
maternal mortality and maternal mortality. It leads to increased morbidity, as well as fetal morbidity and mortality $(6,7)$. Parallel to the increase in the rates of gestational diabetes, gestational hypertension and preeclampsia in women who conceive after ART, a significant increase in the prevalence of preterm labor, low birth weight infants, respiratory distress syndrome and cerebral palsy draws attention (2,6-11). This study was carried out retrospectively in order to draw attention to the increase in maternal, fetal and perinatal mortality and morbidity rates in twin pregnancies due to ART. Spontaneous twin pregnancies were included in the control group, and maternal, fetal and perinatal morbidity and mortality differences were compared with twins due to ART.

\section{METHODS}

This retrospective study was conducted after the approval of the Ethics Committee of Hatay Mustafa Kemal University (approval number: 06, approval date: 05/09/2019). The files of 5,670 pregnant women who admitted to our clinic between 01.01.2011 and 31.12.2017 and gave birth for 20 weeks or later, 500 grams (gr) or more were retrospectively analyzed. Patient consent was obtained in order to use the registered data of the patients. Multiple pregnancy records were made among the pregnant women who gave birth, and the clinical and demographic data of the patients were recorded. Maternal parameters subjected to registration process were; age, form of multiple pregnancy [spontaneous, intrauterine insemination, in vitro fertilization (IVF)], gravida, parity, abortion, number of surviving children, last menstrual period. The fetal parameters recorded were; week of birth, birth weight, mode of delivery, $1^{\text {st }}$ and $5^{\text {th }}$ minutes APGAR scores were determined. When it comes to maternal complications; physiological anemia of pregnancy, preeclampsia and gestational diabetes were taken into consideration. As a fetal complication, preterm birth, premature rupture of membranes, oligo-polyhydramnios, low birth weight baby, birth weight incompatibility, presence of fetal anomaly, fetal death were taken into consideration.

Multiple pregnancies with live births were classified as immature, premature and mature according to the following criteria; births between $20^{\text {th }}$ and $28^{\text {th }}$ weeks were recorded as early preterm (immature) births, births between $28^{\text {th }}$ and $37^{\text {th }}$ weeks as preterm birth (premature), and births at $37^{\text {th }}$ weeks and above as term (mature) births. In the classification made according to fetal birth weight; Babies born 2,500 gr and above were considered as normal birth weight, babies born below 2,500 gr low birth weight. In twin pregnancy deliveries, if there is a difference of $25 \%$ or more in terms of birth weight between twins, it was recorded as discordance. The perinatal mortality index (PMI) was calculated according to the formula below.

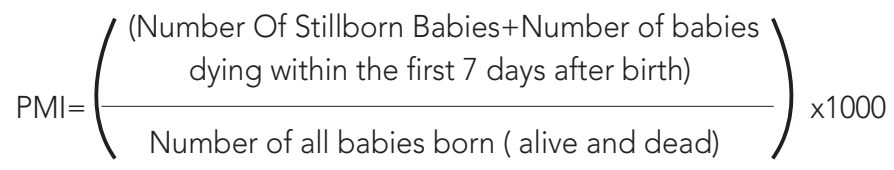

\section{Statistical Analysis}

SPSS 21 (SPSS Inc. Chicago, Illinois, USA) package program was used for statistical analysis of the obtained data. Spontaneous and ART twin births were compared with each other in terms of clinical, demographic, maternal and fetal complications. Student t-test was used for analysis of continuous variables. The obtained results were given as mean \pm standard deviation or as $n$ (\%). Chisquare $\left(\chi^{2}\right)$ tests were used for the analysis of non-continuous variables. $P<0.05$ was considered significant.

\section{RESULTS}

Out of 5,670 pregnant women 102 were deliveries due to multiple pregnancy. Of the 102 multiple births, 94 (92.2\%) were twin and 8 $(7.8 \%)$ were triplet births. Seventy-four of the twins $(78.7 \%)$ were spontaneous twins, 20 of them (21.2\%) were twin pregnancies due to ART. Sixteen of ART twins were obtained due to IVF and 4 of them were due to ovulation induction $(\mathrm{OI})$. Among all pregnant women, the incidence of multiple pregnancy was $1 / 56$, twin pregnancy incidence 1/60, and triplet pregnancy incidence 1/709. In our records, there were no quadruplet and quintet pregnancies that occurred either due to ART or spontaneously.

While the spontaneous twin birth incidence was 1/77, the incidence of twin births due to ART was found to be 1/283.5 (Table 1a). Average maternal age was found to be $29.25 \pm 6.00$ years in general twin pregnancy deliveries, $28.50 \pm 6.09$ years for mothers who had spontaneous twins, and $32.54 \pm 5.09$ years for mothers with twins due to ART. The average age of mothers who gave birth to twins due to ART was found to be higher than mothers who gave birth to twins spontaneously $(p<0.007)$ (Table 1a, Graphic 1). While $9.46 \%$ of spontaneous twins were over 35 years old, $35 \%$ of ART twins were over 35 years old and the difference was found to be significant $(p<0.01)$ (Table 1a, Graphic 2).

Gravida (spontaneous 3.02 $\pm 1.60 ;$ ART 1.64 $\pm 0.84 ; p=0.004$ ), parity (1.72 $\pm 1.30 ; 0.43 \pm 0.76 ; p=0.001)$, mean living child (1.49 \pm 1.30 ; $0.36 \pm 0.74 ; p=0.003)$ in spontaneous twins was found to be

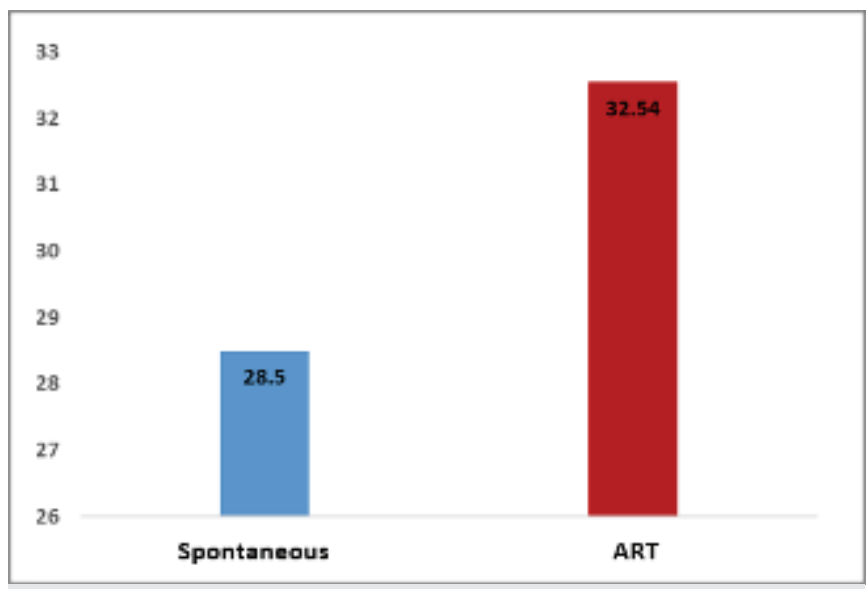

Graphic 1. Average maternal age in spontaneous and ART twin pregnancies

ART: assisted reproductive technology 


\section{Table 1a.Maternal fetal parameters of twin pregnancy deliveries}

\begin{tabular}{|c|c|c|c|c|c|c|}
\hline & $\begin{array}{l}\text { Spontaneous } \\
\text { twins }\end{array}$ & ART twins & \multirow[t]{2}{*}{$p^{*}$} & IVF twins & $\begin{array}{l}\text { Ovulation } \\
\text { induction }\end{array}$ & Total \\
\hline & \multicolumn{2}{|c|}{ n (\%) } & & \multicolumn{3}{|c|}{$n(\%)$} \\
\hline Total birth & 74 (78.72) & $20(21.27)$ & 0.001 & $16(17)$ & $4(4)$ & $94(100)$ \\
\hline$\leq 20$ years old mothers & $9(12)$ & 0 & - & 0 & 0 & $9(9.6)$ \\
\hline$\leq 35$ years & $67(90.54)$ & $13(65)$ & \multirow{2}{*}{0,01} & $9(56)$ & $4(9)$ & 80 \\
\hline Above 35 years old & $7(9.46)$ & $7(35)$ & & $7(44)$ & 0 & 14 \\
\hline Twin birth caesarean & $63(85)$ & $16(80)$ & \multirow{2}{*}{0.731} & $12(75)$ & $4(100)$ & $78(83)$ \\
\hline Twin birth vaginal birth & $11(15)$ & $4(20)$ & & $4(25)$ & 0 & $16(17)$ \\
\hline Live born baby & $140(95)$ & $32(80)$ & 0.004 & $25(78)$ & $7(87.5)$ & $172(91.5)$ \\
\hline Number of babies alive & $136(97)$ & $30(93.75)$ & 0.387 & $23(92)$ & 7 & $166(96.5)$ \\
\hline Incidence & $1.3 / 100$ & $3.52 / 1,000$ & 0.719 & $2.82 / 1,000$ & $7.0 / 10,000$ & $94(1.66)$ \\
\hline Total sectio & & & & & & $4,297(75.8)$ \\
\hline Total vaginal delivery & & & & & & 1,373 (24.2) \\
\hline All births & & & & & & $5,670(100)$ \\
\hline \multicolumn{7}{|l|}{ Maternal parameter } \\
\hline Average maternal age & $28.50 \pm 6.09$ & $32.54 \pm 5.09$ & 0.007 & $33.8 \pm 5.14$ & $28.33 \pm 1.53$ & $29.25 \pm 6$ \\
\hline Gravidite average & $3.02 \pm 1.60$ & $1.64 \pm 0.84$ & 0.004 & $1.45 \pm 0.69$ & $2.33 \pm 1.155$ & $2.54 \pm 1.59$ \\
\hline Parity average & $1.72 \pm 1.30$ & $0.43 \pm 0.76$ & 0.001 & $0.27 \pm 0.65$ & $1 \pm 1$ & $1.25 \pm 1.27$ \\
\hline Abortion & $0.33 \pm 0.76$ & $0.21 \pm 0.43$ & 0.51 & $0.18 \pm 0.40$ & $0.33 \pm 0.58$ & $0.28 \pm 0.69$ \\
\hline Living average & $1.49 \pm 1.30$ & $0.36 \pm 0.74$ & 0.003 & $0.18 \pm 0.60$ & $1 \pm 1$ & $1.14 \pm 1.26$ \\
\hline \multicolumn{7}{|l|}{ Maternal complications } \\
\hline Anemia during pregnancy & $45(61)$ & $14(70)$ & 0.545 & $10(62.5)$ & $4(100)$ & $59(63)$ \\
\hline Preeclampsia-eclampsia & $15(20)$ & $3(15)$ & 0.845 & $3(19)$ & 0 & $18(19.15)$ \\
\hline Gestational diabetes & 1 & & & & & 1 \\
\hline Diabetes mellitus & 1 & & & & & 1 \\
\hline Others & 4 & 1 & - & - & - & 5 \\
\hline EMR & $11(15)$ & $2(10)$ & - & $2(12.5)$ & 0 & $13(14)$ \\
\hline Oligo-anhidroamnios & $5(7)$ & $2(10)$ & - & $2(12.5)$ & 0 & $7(7.4)$ \\
\hline Polyhydramnios & $1(1.35)$ & $1(5)$ & - & $1(6.25)$ & 0 & $2(2.13)$ \\
\hline
\end{tabular}

significantly higher than the ART twins (Table 1a, Graphic 3). The number of babies born alive was found to be significantly higher in spontaneous twin births compared to twin births due to ART (95\%, 80\%, p=0.004, Table 1a, Graphic 4). No significant difference was found between spontaneous and ART twins in terms of abortion rates $(0.33 \pm 0.76$ vs $0.21 \pm 0.43 ; p=0.51)$. When anemia was excluded in all twin pregnancies, maternal morbidity was found as $27 \%$. Anemia was detected in $63 \%$ of the cases who gave birth to twins (61\% spontaneous twin, $70 \%$ due to ART), and $19 \%$ preeclampsia (20\% in spontaneous twins; $15 \%$ in twins due to ART).

While the week of delivery was $35.18 \pm 3.27$ weeks in spontaneous twins, it was found as $34.27 \pm 4.20$ weeks in ART twins (Table 1b,
Graphic 5). When twin births were evaluated in terms of APGAR scores, $1^{\text {st }}$ minute $A P G A R$ was found as $6.30 \pm 1.87$ points in spontaneous twins and 6.21 \pm 2.02 points in ART twins. There was no difference between the groups in terms of $1^{\text {st }}$ minute APGAR values $(p=0.383)$, while $5^{\text {th }}$ minute APGAR values were $8.12 \pm 1.56$ points in spontaneous twins and $7.42 \pm 2.11$ points in twins with ART ( $p=0.103$, Table 1b, Graphic 5).

While the mean birth weight was $2223.87 \pm 603.97 \mathrm{~g}$ in spontaneous twin pregnancies, it was found similar as $2270.50 \pm 811.02 \mathrm{~g}$ in twin pregnancies due to ART ( $p=0.77$ ). While it was $34 \%$ in spontaneous twins who were born 2,500 gr and above, it was found as $62.5 \%$ in ART twins ( $p=0.031$; Table 1b). Low birth weight was found as $53.57 \%$ in spontaneous twin pregnancy deliveries and $18.75 \%$ in 




Graphic 2. Distribution ratio of maternal age below and above 35 years in spontaneous and ART twin pregnancies ART: assisted reproductive technology

ART twins $(p=0.056)$. Discordance between fetuses is shown in Table $1 b$ as SGA, AGA, LGA. Cleft lip, cleft palate, left ventricular cyst in the heart, unilateral polycystic kidney and bilateral ventriculomegaly were found as fetal anomalies. Due to the low number of cases in terms of fetal anomalies, no comparison could be made between the groups.

While both fetuses were stillborn in two of the spontaneous twin pregnancies; In the remaining four deliveries, one of the fetuses was born alive and the other was dead. Both fetuses were stillborn in one of the twin pregnancy deliveries caused by ART. When the gender of stillborn twin babies were also evaluated, the rate of male babies was found to be higher in both spontaneous and ART-induced twins (Table 1b). When all twins were examined, 54\% of the babies were female. The frequency of female babies was found to be higher in spontaneous twins compared to ART twins (61\%; 30\%, respectively; $\mathrm{p}<0.001$; Table $1 \mathrm{~b}$ ).

Fetal mortality rate was $5.4 \%$ in spontaneous twin births and $20 \%$ in ART twins $(p=0.003)$. Neonatal mortality rate was 5.4 in spontaneous twins; and 6.25 in twins connected to ART. PMI was 81 in spontaneous twins and 250 in ART twins $(p<0.006), 117$ in all twins.

\section{DISCUSSION}

In the USA, $11 \%$ of all newborns are reported to occur following the infertility treatment (1). 95\% (1) of all multiple pregnancies in the USA, 99\% (3) in the United Kingdom (UK), and 91\% in Turkey Güler et al. (4) reported to have twin pregnancy births. Of the 102 multiple births in our clinic, 94 (92.2\%) were twins and 8 (7.8\%) were triplets. Our data are consistent with literature data. It has been reported that $18 \%$ of twin births in the USA, 18\% in the UK, $23.3 \%$ in Australia and $41 \%$ in Italy were caused by ART $(1,3,12,13)$. It was found that 74 (78.72\%) of the twins born in our clinic were spontaneous and 20 (21.27\%) developed with ART, our findings were consistent with the literature. Güler et al. (4) reported that $66.6 \%$ of the twins were with ART and $33.4 \%$ were spontaneous. We found that $16(80 \%)$ of ART twin births were caused by IVF and $4(20 \%)$ of them were caused by Ol. Among all pregnant women, the incidence of multiple pregnancy was $102 / 5670(1 / 56)$, twin pregnancy incidence 94/5670 (1/60), and triplet pregnancy incidence $8 / 5670$ (1/709) (Table 1a). Twin births constitute 3-4\% of all births in the USA (1), Bardis et al. (3) reported that $1.45 \%$ of them were twin pregnancy births in Australia. Güler et al. (4) stated the rate of twin birth as $2.7 \%$ in their study. Samani et al. (5) reported the rate of multiple births in Iran as $1.48 \%$. Spontaneous twin birth rate has been reported as $0.6 \%$ to $2.79 \%$ (14), and the rate of twin births among those who underwent ART has been reported as $6.8 \%$ to $8.44 \%(3,5,15)$.

In studies, the average age of mothers who gave birth to twins with ART was found to be higher than the age of mothers who gave birth spontaneously $(3,4,12)$. In Australia, Wang et al. (12) reported that $25.8 \%$ of spontaneous twins are 35 years old and over, and $45.2 \%$ of ART twins are over 35 years old. In our study, $9.46 \%$ of spontaneous twins were over 35 years old, while $35 \%$ of ART twins were over 35 years old ( $p=0.010$, Table 1a, Graphic 2).

In the gravida, parity, living child examination, it is reported in the literature that ART is predominant in mothers who gave birth to primipara twins, and spontaneous twins are dominant in multipara. It has been reported that the number of surviving children of mothers who gave birth to twins spontaneously is higher $(7,10,12,13)$. Gravida, parity, and the mean of living children



Graphic 3. Gravida, parity, living child average in spontaneous and ART twin pregnancies

ART: assisted reproductive technology, avg.: average



Graphic 4. The ratio of live born babies and living babies in spontaneous and ART twin pregnancies ART: assisted reproductive technology 
were found to be higher in spontaneous twins compared to ART twins (Table 1a, Graphic 3, 4). In our study, we obtained findings compatible with the literature.

The rate of preterm birth ( $<37$ weeks) in twin pregnancy deliveries ranges between $36.58 \%$ and $79 \%(2,8,9)$. It has been reported that preterm delivery in ART pregnancies is higher than spontaneous twin pregnancy deliveries due to ART procedure and the underlying reasons $(6,7,12,13,16,17)$. In our study, the week of delivery was $35.18 \pm 3.27$ weeks in spontaneous twins; $34.27 \pm 4.20$ weeks in ART twins, similar to the literature. It has been reported that the live birth rate is lower in ART twin pregnancy births than in spontaneous twin pregnancy births (11-13). In our study, the number and rate of babies born alive were found to be higher in spontaneous twins $(95 \% ; 80 \%$, respectively; $p=0.004$, Table $1 a$, Graphic 4).



Graphic 5. Spontaneous ART birth rates above 37 weeks and below 36 weeks and below 34 weeks in twin pregnancies ART: assisted reproductive technology

In the literature, it has been reported that maternal morbidity and complications are higher in ART pregnancies than in spontaneous pregnancies (11-13). Preeclampsia rate was reported as $19.6 \%$ in ART pregnancies and $13.6 \%$ in spontaneous pregnancies $(8,11,13)$. In twin pregnancy deliveries, when anemia was excluded, we found maternal morbidity $27 \%$, anemia $63 \%$ and preeclampsia $19 \%$. No significant difference could be found between maternal complications in spontaneous twin pregnancy delivery and ART twin pregnancy deliveries, this may be due to the low number of our cases.

There are publications reporting that baby birth weight is significantly higher than spontaneous twin pregnancy births in ART-induced twin pregnancy deliveries $(3,11)$ and there are also publications reporting that there is no difference $(4,6,12,13)$. There was no significant difference between the groups in terms of birth weight. In spontaneous twins, births of 2,500 gr and above were found to be $34 \%$; It was $62.5 \%$ in ART twins ( $p=0.031$; Table $1 b$ ). The reason may be that mothers who were pregnant with ART had better perinatal follow-up than mothers who were spontaneously pregnant. There was no significant difference between the groups in terms of the presence of discordance between fetuses. Comparison could not be made in terms of fetal anomalies due to the low number of cases.

There are publications (13) reporting that APGAR $1^{\text {st }} 5^{\text {th }}$ minute scores are higher (4) or no difference (12) in spontaneous twin pregnancy deliveries. We also did not find any difference between $1^{\text {st }} 5^{\text {th }}$ minutes APGAR values (Table $1 \mathrm{~b}$ ).

There was no significant difference between the groups in terms of the sex of twin babies born alive. When the gender of stillborn twin babies was evaluated, male baby gender was found to be $81.25 \%$ (Table 1b). This is because in fetal life, female sex fetuses are more prone to survival than male sex fetuses. Özkan et al. (18) reported male gender as $54 \%$ in his study on fetal autopsies. When all twins were examined, $57.5 \%$ of the babies were female babies. Yumru et al. (19) reported the male baby gender at birth as $52 \%$. In our study, the frequency of female babies was found to be higher in spontaneous twins compared to ART twins (spontaneous $60.7 \%$; ART 44\%).

While neonatal death was reported as $2.1 \%$ in ART pregnancies in twins; this rate is $1.8 \%$ in spontaneous pregnancies (12). In our study, neonatal mortality was $5.4 \%$ in spontaneous twins; $6.25 \%$ of twins born with ART; fetal mortality was $5.4 \%$ in spontaneous twin pregnancy and $20 \%$ in ART twins $(p=0.003)$. In singleton, twin and multiple pregnancy deliveries resulting from ART; it has been reported that perinatal mortality and morbidity are higher than spontaneous pregnancy deliveries $(3,6,12,13)$. We found PMI as 117 in all twins, 81 in spontaneous twins, and 250 in ART twins. Our findings are consistent with the literature data.

\section{Study Limitations}

The limitations of our study are the retrospective nature of our study and the low number of our cases as 94 . We hope that the studies on a much larger number of cases will contribute significantly to this issue.

\section{CONCLUSION}

We can list the measures that can be taken to reduce the maternal and fetal negative effects of ART-related multiple pregnancies as follows: Single embryo transfer should be done in IVF. In cases where 3 or more follicles develop during $\mathrm{OI}$ and intrauterin insemination, IVF application or cycle cancellation should be performed. In IVF cycles, ovarian stimulation should be done with low dose gonadotropin. Embryo cryopreservation is required for cycles with hyperstimulation in IVF. While the patient is preparing for IVF, estradiol level should be kept below $3,000 \mathrm{pg} / \mathrm{mL}$ as much as possible. We can reduce the occurrence of multiple pregnancies due to ART by using analogues instead of human chorionic gonadotropin and embryo cryopreservation in triggering ovulation in cases where estradiol level is $3,000 \mathrm{pg} / \mathrm{mL} \leq$. 


\section{Table 1b. Fetal parameters of twin pregnancy deliveries}

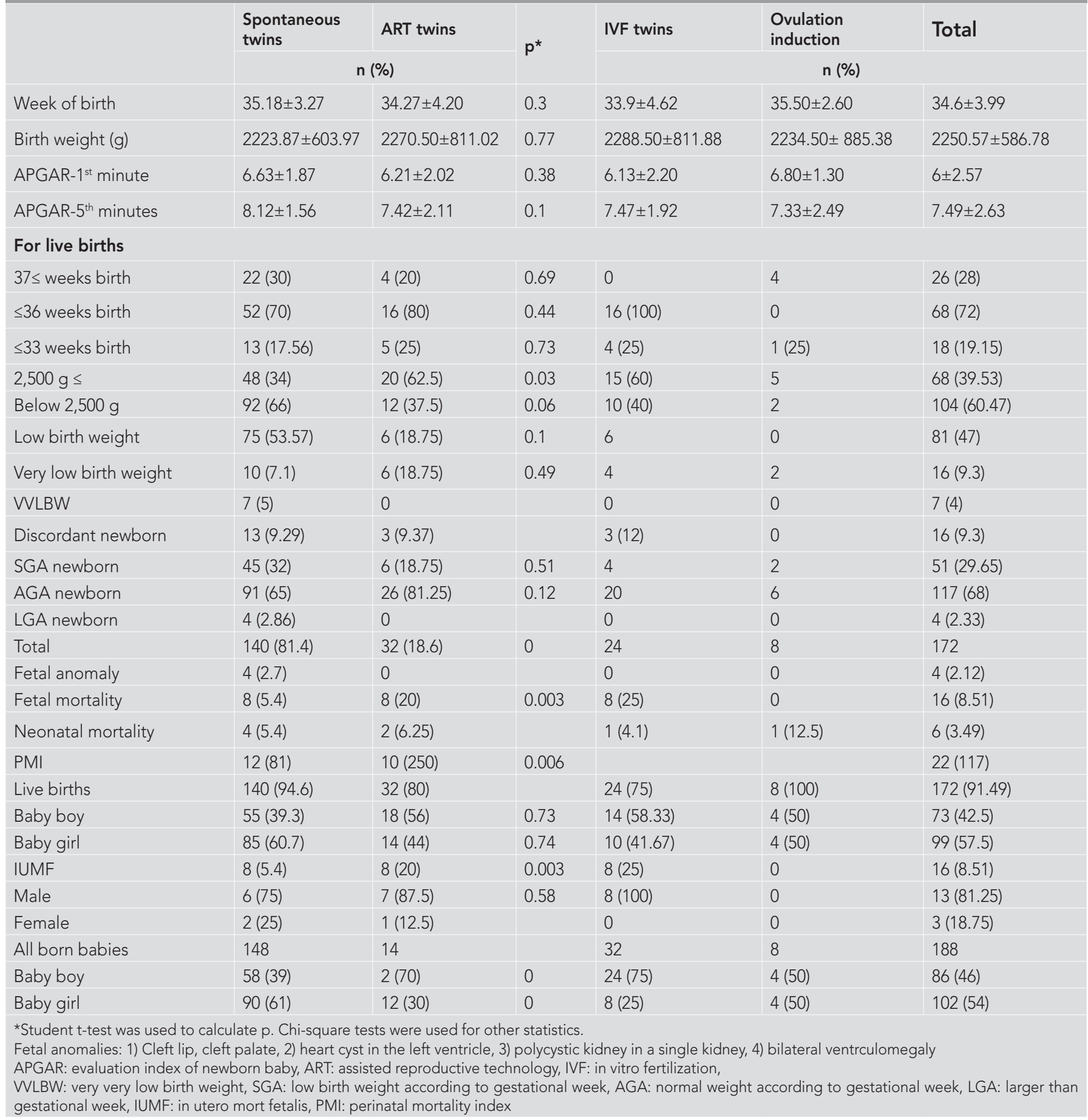

Ethics Committee Approval: This retrospective study was conducted after the approval of the Ethics Committee of Hatay Mustafa Kemal University (approval number: 06, approval date: 05/09/2019).

Informed Consent: Patient consent was obtained in order to use the registered data of the patients.

Peer-review: Externally peer-reviewed.

Financial Disclosure: The author declared that this study has received no financial support.

\section{REFERENCES}

1. Ananth CV, Chauhan SP. Epidemiology of twinning in developed countries. Semin Perinatol 2012; 36: 156-61.

2. Yelland LN, Schuit E, Zamora J, Middleton PF, Lim AC, Nassar AH, et al. Correlation between neonatal outcomes of twins depends on the outcome: secondary analysis of twelve randomised controlled trials. BJOG 2018; 125: 1406-13.

3. Bardis N, Maruthini D, Balen AH. Modes of conception and multiple pregnancy: a national survey of babies born during one week in 2003 in the United Kingdom. Fertil Steril 2005; 84: 1727-32. 
population-based retrospective cohort study. BMC Pregnancy Childbirth 2018; 18: 320 .

13. Bordi G, D'Ambrosio A, Gallotta I, Di Benedetto L, Frega A, Torcia F, et al. The influence of ovulation induction and assisted conception on maternal and perinatal outcomes of twin pregnancies. Euro Rev Med Pharmacol Sci 2017; 21: 3998-4006.

14. Smits J, Monden C. Twinning across the developing world. PLoS One 2011; 6: e25239. doi: 10.1371/journal.pone.0025239.

15. Macaldowie A, Lee E, Chambers G. Assisted reproductive technology in Australia and New Zealand 2013. Sydney: National Perinatal Epidemiology and Statistics Unit, the University of New South Wales; 2015.

16. Thomson F, Shanbhag S, Templeton A, Bhattacharya S. Obstetric outcome in women with subfertility. BJOG 2005; 112: 632-7.

17. Farhi J, Haroush AB, Andrawus N, Pinkas H, Sapir O, Fisch B, et al. High serum oestradiol concentrations in IVF cycles increase the risk of pregnancy complications related to abnormal placentation. Reprod Biomed Online 2010; 21: 331-7.

18. Özkan F, Cesur S, Bilgiç $R$, Yorgancı C. Retrospective analysis of our fetal autopsies in a period of 13 years. Türk Pediatri Arşivi 2006; 41: 46-50.

19. Yumru AE, Dinçgez B, Öndeş B, Atalay N. The relation between gender of babies, macrosomia and cesarean ratio in 11561 deliveries in our clinic. JAREM 2011; 1: 18-20. 